\title{
QT interval and antidepressant use: a cross sectional study of electronic health records
}

\author{
(c) $)(1)$ (8)
}

\author{
Victor M Castro team lead ${ }^{1}$, Caitlin C Clements clinical research coordinator ${ }^{23}$, Shawn N Murphy \\ associate professor of neurology ${ }^{4}$, Vivian S Gainer team lead ${ }^{1}$, Maurizio Fava Slater Family professor \\ of psychiatry $^{5}$, Jeffrey B Weilburg assistant professor of psychiatry ${ }^{5}$, Jane L Erb assistant professor \\ of psychiatry ${ }^{6}$, Susanne E Churchill executive director, i2b2 National Center for Biomedical \\ Computing $^{7}$, Isaac S Kohane director, i2b2 National Center for Biomedical Computing ${ }^{8}$, Dan V \\ losifescu associate professor of psychiatry ${ }^{9}$, Jordan W Smoller associate professor of psychiatry ${ }^{2}$, \\ Roy H Perlis associate professor of psychiatry ${ }^{3}$
}

\begin{abstract}
${ }^{1}$ Partners Research Computing, Partners HealthCare System, One Constitution Center, Boston, MA 02129, USA; ${ }^{2}$ Psychiatric and Neurodevelopmental Genetics Unit, Department of Psychiatry, Massachusetts General Hospital, Boston, MA 02114, USA; ${ }^{3}$ Center for Experimental Drugs and Diagnostics, Department of Psychiatry, Massachusetts General Hospital, Boston, MA 02114, USA; ${ }^{4}$ Laboratory of Computer Science and Department of Neurology, Massachusetts General Hospital, Boston, MA 02114, USA; ${ }^{5}$ Depression Clinic and Research Program, Department of Psychiatry, Massachusetts General Hospital, Boston, MA 02114, USA; ${ }^{6}$ Department of Psychiatry, Brigham and Women's Hospital, Boston, MA 02215, USA; ${ }^{7}$ Information Systems, Partners HealthCare System, New Research Building 255, Boston, MA 02215, USA; ${ }^{8}$ Department of Medicine, Brigham and Women's Hospital, Boston, MA 02215, USA; ${ }^{9}$ Mood and Anxiety Disorders Program, Department of Psychiatry, Mount Sinai School of Medicine, New York, NY 10029, USA
\end{abstract}

\begin{abstract}
Objective To quantify the impact of citalopram and other selective serotonin reuptake inhibitors on corrected QT interval (QTC), a marker of risk for ventricular arrhythmia, in a large and diverse clinical population.

Design A cross sectional study using electrocardiographic, prescribing, and clinical data from electronic health records to explore the relation between antidepressant dose and QTC. Methadone, an opioid known to prolong QT, was included to demonstrate assay sensitivity.

Setting A large New England healthcare system comprising two academic medical centres and outpatient clinics.

Participants 38397 adult patients with an electrocardiogram recorded after prescription of antidepressant or methadone between February 1990 and August 2011.
\end{abstract}

Main outcome measures Relation between antidepressant dose and QTc interval in linear regression, adjusting for potential clinical and demographic confounding variables. For a subset of patients, change in QTC after drug dose was also examined.

Results Dose-response association with QTc prolongation was identified for citalopram (adjusted beta 0.10 (SE 0.04), $\mathrm{P}<0.01$ ), escitalopram (adjusted beta $0.58(0.15), P<0.001)$, and amitriptyline (adjusted beta
0.11 (0.03), $\mathrm{P}<0.001$ ), but not for other antidepressants examined. An association with QTc shortening was identified for bupropion (adjusted beta $0.02(0.01) \mathrm{P}<0.05)$. Within-subject paired observations supported the QTc prolonging effect of citalopram (10 $\mathrm{mg}$ to $20 \mathrm{mg}$, mean QTc increase 7.8 (SE 3.6) ms, adjusted $\mathrm{P}<0.05$; and $20 \mathrm{mg}$ to $40 \mathrm{mg}$, mean QTc increase 10.3 (4.0) ms, adjusted $\mathrm{P}<0.01$ ).

Conclusions This study confirmed a modest prolongation of QT interval with citalopram, and identified additional antidepressants with similar observed risk. Pharmacovigilance studies using electronic health record data may be a useful method of identifying potential risk associated with treatments.

\section{Introduction}

Fatal arrhythmias, including torsades de pointes, may be precipitated by prolongation of ventricular repolarisation caused by medication. Numerous marketed drugs have been associated with effects on repolarisation interval, characterised by lengthening of the QT interval on an electrocardiogram; some drugs were withdrawn from the market when such lengthening was identified in postmarketing surveillance. ${ }^{1}$ Several commonly used psychotropic medications, including some antidepressants, 
have been reported to prolong QT interval. ${ }^{2-4}$ Because incidence of torsades de pointes is extremely low, QT prolongation is widely used as a surrogate marker of risk, albeit an imperfect one. $^{5}$

In August 2011, the US Food and Drug Administration (FDA) announced that the selective serotonin reuptake inhibitor citalopram had been associated with QT prolongation at higher doses, informing clinicians that "Citalopram causes dose-dependent QT interval prolongation. Citalopram should no longer be prescribed at doses greater than $40 \mathrm{mg}$ per day." Further clarification issued in March 2012 restricted the maximum dose to $20 \mathrm{mg}$ for subgroups of patients, including those older than 60 years and those taking an inhibitor of cytochrome $\mathrm{P} 4502 \mathrm{C} 19.7$ This change, affecting the most widely prescribed antidepressant in the US, with 37.8 million prescriptions in $2011,{ }^{8}$ left clinicians unclear about appropriate next-step strategies because of the lack of data comparing citalopram with other antidepressants.

Traditionally, QT interval is examined in registration trials and postmarketing surveillance, both of which have substantial limitations. The former may sample a restricted group of patients and may not be reflective of the medical comorbidities or concomitant treatments that may increase risk of QT prolongation. The latter captures only severe or unexpected outcomes, does not do so consistently, and may be slow to detect rare outcomes. ${ }^{9}$

To address these limitations and to better understand the extent of QT prolongation in a general clinical population, we used a pharmacovigilance approach, which applied natural language processing and machine-learning algorithms to examine electronic health records from a large New England healthcare system encompassing more than four million individuals. This approach has been shown to be valid and sensitive to adverse cardiovascular outcomes in multiple investigations. ${ }^{10-12}$ Here, we examined QT intervals in individuals prescribed antidepressants, as well as in individuals prescribed the opioid methadone, known to be a contributor to QT prolongation and included as a measure of assay sensitivity.

\section{Methods \\ Overview and dataset generation}

The Partners HealthCare electronic health record includes sociodemographic data, billing codes, laboratory results, problem lists, medications, vital signs, procedure reports, and narrative notes from Massachusetts General Hospital and Brigham and Women's Hospital, as well as from community and specialty hospitals that are part of the Partners HealthCare system in Boston (Massachusetts, USA). Adult patients (age $\geq 18$ years) with at least one prescription of an antidepressant or methadone between February 1990 and August 2011 were selected from the electronic health record for inclusion in a dataset (referred to as a data "mart"), yielding 241308 subjects from about four million unique patients from the Massachusetts General Hospital and Brigham and Women's Hospital systems. The data mart consists of all electronic records (psychiatric and non-psychiatric) and can be managed with the $\mathrm{i} 2 \mathrm{~b} 2$ server software (i2b2 version 1.5, Boston, MA, USA). ${ }^{13}$ The i2b2 system is a scalable computational framework, deployed at over 60 major academic health centres, for managing human health data. ${ }^{14}{ }^{15}$ The Partners Institutional Review Board approved all aspects of this study.

Medications are documented from drugs prescribed to patients in the outpatient electronic health record $(55 \%)$ and drugs dispensed by the inpatient pharmacy (45\%). All prescriptions were provided with a supply ranging from 30 to 180 days, including refills. Confirmation that drugs prescribed were actually dispensed to patients is not available for research purposes by agreement between the hospitals and the pharmacy data provider. The present analysis included the antidepressants citalopram, escitalopram, fluoxetine, paroxetine, sertraline, amitriptyline, bupropion, duloxetine, mirtazapine, nortriptyline, and venlafaxine, as well as the opioid methadone.

Electrocardiographic reports for study patients were parsed by text pattern matching algorithms to extract QTc measurements, then normalised to standardised units. A manual review of 200 electrocardiographic reports showed $100 \%$ concordance with extracted values. While differences exist between automated QTc estimation algorithms, these values generally correlate well with both semi-automated and manual measurements by cardiologists. ${ }^{16}$ Correlation is poorer among individuals with pre-existing arrhythmias, so ventricular arrhythmia was included as a covariate in all analyses. Year of electrocardiography was also included to address changes in electrocardiographic algorithms and software over time.

For primary analysis, we identified individuals treated with at least one medication of interest, and selected the most recent electrocardiogram (within 90 days) occurring at least 14 days after a prescription. The lower threshold was selected to allow time for individuals to fill the prescription, and to ensure that the drug had reached steady state at the new dose. Where multiple electrocardiograms for a given patient were within this time window, we selected the one corresponding to the maximum drug dose in order to enrich sampling of the high end of the dose range.

Three sensitivity analyses were conducted to assess the assumptions of antidepressant exposure in the study design. We first excluded individuals who did not have a follow-up prescription after their initial prescription, and subsequently included only QTc measurements obtained 14-30 days after prescription. To further assess the impact of concomitant medications on our analysis, we also excluded individuals with prescriptions of typical or atypical antipsychotics or known QT prolongers astemizole, cisapride, or methadone within one year of the electrocardiogram.

\section{Analysis}

We first tested QTc as a continuous measure for evidence of association with drug dose. Linear regression models included dose as well as patient's age, sex, public versus private health insurance, and self reported ethnicity. (In this health system, Hispanic or Latino race is not captured separately from ethnicity, so subjects self reporting as Hispanic are analysed as a distinct group.) Covariates were included to examine potential confounding effects of cardiovascular disease history including history of myocardial infarction, ventricular fibrillation, hypertension, or hyperlipidaemia, and log transformed, age adjusted Charlson comorbidity score. ${ }^{17}{ }^{18}$ Additional covariates for presence or absence of methadone and presence or absence of a first generation antipsychotic were also examined. A diagnosis of major depressive disorder versus all other psychiatric disorders was also included in the model. Covariate association with QTc was assessed using univariate linear regression. Where the dose effect was significant in linear regression models, post hoc pairwise comparisons were used to examine threshold effects (that is, whether specific increments contributed to QT prolongation). 
Where the linear dose term was nominally significant at $\mathrm{P}<0.05$, we also used a complementary means of analysis to examine within-subject QTc effects. For a subset of individuals, electrocardiograms were available at two escalating doses (for example, after documentation of citalopram $20 \mathrm{mg}$ and of citalopram $40 \mathrm{mg}$ ), enabling an analysis similar to a traditional crossover design. The second dose was restricted to less than two years from the initial dose. For this subset of patients, we examined mean change in QT as an estimate of dose response. We used the paired $t$ test to test differences between initial and subsequent increased dose per patient. Escalating doses with fewer than 10 patients were excluded from this analysis.

Finally, for descriptive purposes, we examined the proportion of subjects in QTc prolongation categories previously applied in the literature. ${ }^{19}$ QTc values were characterised as normal, borderline, abnormal, or high, based on published thresholds (normal is $\leq 430 \mathrm{~ms}$ for men, $\leq 450 \mathrm{~ms}$ for women; borderline 431-450 ms for men, 451-470 ms for women; abnormal 451-500 ms for men, 471-500 ms for women; high >500 ms for men and women). ${ }^{19}$

For all analyses, we used R 2.13 (R Foundation for Statistical Computing, Vienna, Austria).

\section{Results}

A total of 1472965 valid QTc measurements were extracted from 1497333 electrocardiograms (98.4\%). Of the study patients receiving a prescription of interest, $38397 \mathrm{had}$ an electrocardiogram within the 14-90 day window. Characteristics of this cohort and the proportion receiving each medication are summarised in table $1 \Downarrow$. Table 1 also indicates association between each sociodemographic and clinical feature and QTc interval. Age, race, sex, insurance, year of electrocardiogram, history of major depression, history of myocardial infarction, history of ventricular arrhythmia, history of hypertension, history of hyperlipidaemia, and Charlson comorbidity score were all significantly associated with QTc interval $(\mathrm{P}<0.01)$.

Patients prescribed a medication of interest but with no QTc measurements in the exposure period were compared with those with available electrocardiograms to assess selection bias (see table A in supplementary material on bmj.com). As expected, those with an electrocardiogram in the exposure period included in the study cohort were in general older, with greater medical comorbidity and use of healthcare services than patients excluded from the study cohort because no electrocardiogram was available.

Standard thresholds at which QTc prolongation is associated with elevated risk for arrhythmia have been reported. ${ }^{19}$ To allow comparison with these reports, individuals with available electrocardiogram data were categorised according to those thresholds, which differ by sex. Supplementary fig B on bmj.com illustrates the proportion of each cohort with QTc characterised as normal, borderline, abnormal, and high. Of note, in this population $20.4 \%$ of individuals were characterised as having abnormal or high QTc values; these proportions were similar across treatment groups.

For each medication of interest, dose-QTc curves were plotted (figure $\Downarrow$ ) and tested for dose-response relation using a linear model adjusted for features associated with QTc (table $1 \Downarrow$ ). Dose was found to be a significant predictor of QTc in citalopram (adjusted beta 0.10 (SE 0.04) $\mathrm{P}<0.01$ ), escitalopram (adjusted beta $0.58(0.15), \mathrm{P}<0.001)$, and amitriptyline (adjusted beta 0.11 (0.03), $\mathrm{P}<0.001)$. As expected, increasing methadone dose was also associated with increased QTc (adjusted beta 0.30
(0.06), $\mathrm{P}<0.001)$. Increasing doses of bupropion were found to significantly decrease QTc (adjusted beta $0.02(0.01), \mathrm{P}<0.05)$. Where dose was a significant predictor of QTc (table $2 \Downarrow$ ), post hoc pairwise tests compared each dose interval (table $3 \Downarrow$ ). The figure $\Downarrow$ also indicates doses significantly different from the previous dose. Escalating doses with significant increases in QTc were observed in citalopram $10 \mathrm{mg}$ to $20 \mathrm{mg}$ (adjusted beta 9.8 (SE 1.6), $\mathrm{P}<0.001$ ), citalopram $40 \mathrm{mg}$ to $60 \mathrm{mg}$ (adjusted beta $6.1(2.1), \mathrm{P}<0.01$ ), escitalopram $5 \mathrm{mg}$ to $10 \mathrm{mg}$ (adjusted beta 11.0 (4.5), $\mathrm{P}<0.05$ ), escitalopram $10 \mathrm{mg}$ to 20 mg (adjusted beta 4.7 (1.6), $\mathrm{P}<0.01$ ), and amitriptyline $25 \mathrm{mg}$ to $50 \mathrm{mg}$ (adjusted beta $3.4(1.4), \mathrm{P}<0.05)$. Sensitivity analyses restricting individuals based on refill status, exposure window and concomitant medications did not meaningfully alter the results (supplementary tables B, C, and D on bmj.com).

In the within-subject crossover dose analysis, QTc was characterised before and after dose increase in 467 subjects for whom electrocardiogram data were available for multiple doses of citalopram, escitalopram, amitriptyline, bupropion, and methadone. Statistically significant increases in QTc were observed in 59 patients taking citalopram with daily dose increase from $10 \mathrm{mg}$ to $20 \mathrm{mg}$ (mean QTc increase 7.8 (SE 3.6) $\mathrm{ms}$, adjusted $\mathrm{P}<0.05)$ and in 107 patients with citalopram increasing from $20 \mathrm{mg}$ to $40 \mathrm{mg}$ (mean QTc increase 10.3 (4.0) $\mathrm{ms}$, adjusted $\mathrm{P}<0.01$ ) but not in other dose increases (table $4 \Downarrow$ ). We also observed a significant decrease in QTc in 13 patients with an increased daily dose for bupropion from $100 \mathrm{mg}$ to 200 mg (mean QTc decrease 19.2 (8.7) ms, adjusted $\mathrm{P}<0.05$ ). Of note, $13.1 \%$ of patients who started taking citalopram with a QTc in the normal range shifted to "abnormal" after dose increase.

\section{Discussion}

In this pharmacovigilance study using health records from 38 397 patients treated with antidepressants, we identified statistically significant evidence of modest QT prolongation for some pharmacotherapies, namely methadone and the tricyclic antidepressant amitriptyline, as well as for citalopram and escitalopram. However, the sizes of these effects were small, and the proportions of individuals with abnormal QTc intervals were broadly similar across individual antidepressant treatments. Results from a complementary, within-subject means of analysis for citalopram supported a dose-response association. Both sets of results extend a recent announcement from the US Food and Drug Administration (FDA), which was based largely on a small cohort of healthy subjects participating in a crossover study with citalopram. In particular, our results show that several of the antidepressants may be associated individually with lengthening of QT intervals with increasing dose.

\section{Comparison with other studies}

The present results complement previous cohort studies which examined ventricular arrhythmias directly. ${ }^{20}$ Most notably, a recent investigation of Medicaid claims data suggested elevated risk with mirtazapine and diminished risk with bupropion, with hazards otherwise similar across antidepressants. ${ }^{20}$ Although we do not observe strong evidence of dose-response for mirtazapine, we do note shortening of the QT interval with bupropion, which might explain its protective effect in that study, and which previously had been suggested by smaller studies. ${ }^{21} 22$ Also of note, we observe significant QTc prolongation with amitriptyline but not with its metabolite nortriptyline, consistent with a recent preclinical study which identified minimal QTc effects with nortriptyline. ${ }^{23}$ 


\section{Implications of study}

The implications for clinicians merit careful consideration. One notable finding is that nearly one in five patients treated with these antidepressants who underwent electrocardiography had QT intervals which would be considered abnormal. The clinical significance of this prolongation, including the risk of induction of torsades de pointes, is unknown. However, the incidence of torsades de pointes is low among general clinical populations ${ }^{24} 25$ and is similar among patients treated with antidepressants. ${ }^{20}$ For this reason, QTc is often used as a proxy measure for risk of torsades associated with medication, recognising that it is an imperfect surrogate. ${ }^{5}$ Indeed, the FDA warning for citalopram was issued in spite of the epidemiological data showing no difference in risk for arrhythmia. ${ }^{20}$

The question of whether patients for whom antidepressants will be prescribed should routinely have electrocardiograms before and/or after treatment starts cannot be addressed directly by this study. However, in terms of treatment selection, our results do suggest some variation within treatment class in terms of risk. For example, selective serotonin reuptake inhibitors such as sertraline may be associated with less risk than citalopram, and therefore might be preferred in individuals with other risk factors. For escitalopram, our data are less definitive, but suggest that some risk may need to be considered for this drug as well. Of interest, bupropion is routinely used as an augmentation strategy when initial antidepressant treatment fails to achieve remission, though it is not approved by the FDA for this indication. ${ }^{26}$ Our results suggest that, given its capacity to shorten QT interval, bupropion treatment might be a reasonable next step for patients partially responsive to citalopram who would otherwise require a dose increase.

\section{Value of pharmacovigilance studies using data from electronic health records}

Multiple strategies exist for characterising the potential QT effects of pharmacotherapies. In vitro, new compounds are screened for antagonism of the delayed rectifier potassium channel, encoded by hERG (human ether-a-go-go related gene). ${ }^{27}$ Such effects are highly sensitive (though not specific) for risk of torsades de pointes. In light of well publicised withdrawals of compounds from the US market, ${ }^{1}$ drugs with high risk of QT prolongation in such assays may simply not be progressed into human trials. In registration trials, pre-treatment and post-treatment electrocardiograms may be assessed to determine QT effects, and regulatory bodies may request additional studies ${ }^{6}$ to clarify this risk. Although this approach may be useful for demonstrating group effects, it may not be sensitive to QT prolongation that occurs in particular patient subgroups or in a small number of individuals. To complement such studies, postmarketing surveillance allows detection of unanticipated events such as torsades de pointes, though such surveillance depends on voluntary reporting by clinicians of events and the ability to infer association with a particular treatment.

More recently, methods have been developed that allow rapid screening of electronic health records. This approach offers two key advantages. First, it may detect subtle changes that would not have been detected in postmarketing surveillance—such as modest increases in QT interval which would not otherwise be reported. Second, because medical records include a more generalisable population than randomised trials, analysis of electronic health record data may identify risk in patients taking multiple medications or with concomitant medical disorders. For a drug that has already been marketed, this method may be the only feasible way to systematically examine electrocardiographic changes without requiring a crossover trial. Our report builds on previous pharmacovigilance studies of more complex outcomes such as cardiovascular disease, cerebrovascular disease, and gastrointestinal bleeding..$^{11}$

\section{Strengths and limitations of study}

A major strength of the traditional pharmacoepidemiological approach is its focus on "hard" end points of greatest clinical concern; in contrast, QT interval is a strong but imperfect predictor of torsades risk. ${ }^{5}$ Conversely, QT changes may be more sensitive to subtle drug effects (those which modestly increase risk), and a previous report suggested that clinical databases may have limited sensitivity for arrhythmias that do not occur in the inpatient setting. ${ }^{28}$ The use of medical records data also allows further adjustment for potentially confounding clinical variables, which may explain why we detected dose-response effects when prior investigation did not. ${ }^{20}$

A key limitation of our study, which must be considered in interpreting these results, is the lack of random assignment of treatment and dose, which increases risk for confounding. In particular, clinicians may make treatment decisions based on factors not assessed here, and these factors may confound the observed effects on electrocardiographic parameters. For example, if clinicians tended to prescribe sertraline to patients who had experienced myocardial infarction on the basis of the SADHART or SADHART-CHF trials, ${ }^{29}{ }^{30}$ a spurious association between sertraline and QT prolongation might be observed. However, we note that this effect was not observed, that we adjusted for history of myocardial infarction, and that this limitation is also present in traditional postmarketing surveillance studies. Our analyses also relied on data collected before dissemination of the FDA warning for citalopram, which should preclude any impact on citalopram prescribing.

More generally, as with any investigation based on electronic health records, a potential source of bias is the non-random ascertainment of electrocardiograms-most patients treated with antidepressants do not receive electrocardiograms. This should bias results toward greater mean QTc (for example, because of greater age and pre-existing cardiovascular morbidity in those patients who do receive an electrocardiogram), and it probably explains the high prevalence of QTc prolongation overall. Our results may be seen as most relevant to an older, sicker population than the average outpatient cohort treated with antidepressants; indeed, these features correspond to some of the risk factors noted in the FDA warning.

If anything, non-random ascertainment might be anticipated to diminish evidence of a dose-response relation if clinicians avoid dose escalation in patients with QTc prolongation. On the other hand, this systematic bias should exist for all the drugs. This substantial limitation must be balanced against the advantages of rapid characterisation of a large and clinically representative cohort in which potentially confounding clinical variables are readily identified. Still, where a signal of risk is detected, randomised investigation may be required to confirm association.

\section{Conclusions}

Taken together, the present findings demonstrate the potential advantages of using electronic health records for postmarketing surveillance. While it cannot replace careful investigation of QT effects in phase II and III clinical trials, use of electronic health record data provides a useful complement, particularly in terms of comparison with other available interventions. 


\section{What is already known on this topic}

The US Food and Drug Administration has issued warnings that citalopram had been associated with QT prolongation, based on postmarketing surveillance and a small crossover study

No studies have explored this risk in general clinical populations, or its specificity for citalopram

\section{What this study adds}

This study of clinical data from electronic health records suggests a dose-response association with increased QTc interval for citalopram as well as escitalopram and amitriptyline, and decreased QTc for bupropion, though the absolute magnitude of these effects is modest In this analysis, other standard antidepressants were not associated with QTc prolongation

Contributors: VMC designed the tools for collecting data, cleaned and analysed the data, and drafted and revised the manuscript. CCC contributed to preparation of the manuscript. SNM designed the tools for collecting data and contributed to interpretation of analysis and preparation of the manuscript. VSG, MF, JBW, JLE, SEC, ISK, and DVI contributed to interpretation of the analysis and preparation of the manuscript. JWS designed the study and contributed to interpretation of the analysis and preparation of the manuscript. RHP initiated the project, designed the study, monitored the analyses and drafted and revised the manuscript. VMC and RHP are guarantors for the study. All authors had full access to all of the data (including statistical reports and tables) in the study and can take responsibility for the integrity of the data and the accuracy of the data analysis.

Funding: The project was supported by the $\mathrm{NIH} /$ National Library of Medicine (award No 2U54LM008748 to ISK) and the National Institute of Mental Health (award No R01MH086026 to RHP). The content is solely the responsibility of the authors and does not necessarily represent the official views of the National Library of Medicine or the National Institutes of Health.

Competing interests: All authors have completed the ICMJE uniform disclosure form at www.icmje.org/coi_disclosure.pdf (available on request from the corresponding author) and declare: RHP has received consulting fees from or served on scientific advisory boards for Proteus Biomedical, Pamlab, Genomind, and RIDventures, and has received research grant support from Proteus Biomedical and royalties from Concordant Rater Systems. JWS has served as a consultant for the Medical Letter. DVI has received payment for lectures including service on speakers' bureaus from the Massachusetts General Hospital Psychiatry Academy and has served as a consultant for CNS Response. MF has received consulting fees from or served on scientific advisor boards for Abbott Laboratories, Affectis Pharmaceuticals AG, Alkermes, Amarin Pharma, Aspect Medical Systems, AstraZeneca, Auspex Pharmaceuticals, Bayer AG, Best Practice Project Management, BioMarin Pharmaceuticals, Biovail Corporation, BrainCells, Bristol-Myers Squibb, CeNeRx BioPharma, Cephalon, CNS Response, Compellis Pharmaceuticals, Cypress Pharmaceutical, DiagnoSearch Life Sciences, Dinippon Sumitomo Pharma, Dov Pharmaceuticals, Edgemont Pharmaceuticals, Eisai, Eli Lilly, EnVivo Pharmaceuticals, ePharmaSolutions, EPIX Pharmaceuticals, Euthymics Bioscience, Fabre-Kramer Pharmaceuticals, Forest Pharmaceuticals, GenOmind, GlaxoSmithKline, Grunenthal GmbH, i3 Innovus/Ingenis, Janssen Pharmaceutica, Jazz Pharmaceuticals, Johnson \& Johnson Pharmaceutical Research \& Development, Knoll Pharmaceuticals, Labopharm, Lorex Pharmaceuticals, Lundbeck, MedAvante, Merck, MSI Methylation Sciences, Naurex, Neuralstem, Neuronetics, NextWave Pharmaceuticals, Novartis AG, Nutrition 21, Orexigen Therapeutics, Organon Pharmaceuticals, Otsuka Pharmaceuticals, Pamlab, Pfizer, PharmaStar, Pharmavite, PharmoRx Therapeutics, Precision Human Biolaboratory, Prexa Pharmaceuticals, Puretech Ventures,

PsychoGenics, Psylin Neurosciences, Rexahn Pharmaceuticals, Ridge Diagnostics, Roche, Sanofi-Aventis US, Sepracor, Servier Laboratories, Schering-Plough, Solvay Pharmaceuticals, Somaxon Pharmaceuticals, Somerset Pharmaceuticals, Sunovion Pharmaceuticals, Supernus Pharmaceuticals, Synthelabo, Takeda Pharmaceutical, Tal Medical,
Tetragenex Pharmaceuticals, TransForm Pharmaceuticals, Transcept Pharmaceuticals, Vanda Pharmaceuticals. MF has equity holdings in Compellis and has received research grant support from Abbot Laboratories, Alkermes, Aspect Medical Systems, AstraZeneca, BioResearch, BrainCells, Bristol-Myers Squibb, CeNeRx BioPharma, Cephalon, Clintara, Covance, Covidien, Eli Lilly, EIMindA, EnVivo Pharmaceuticals, Euthymics Bioscience, Forest Pharmaceuticals, Ganeden Biotech, GlaxoSmithKline, Icon Clinical Research, i3 Innovus/Ingenix, Johnson \& Johnson Pharmaceutical Research \& Development, Lichtwer Pharma GmbH, Lorex Pharmaceuticals, National Alliance for Research on Schizophrenia \& Depression (NARSAD), National Center for Complementary and Alternative Medicine (NCCAM), National Institute of Drug Abuse (NIDA), National Institute of Mental Health (NIMH), Novartis AG, Organon Pharmaceuticals, PamLab, Pfizer, Pharmavite, Photothera, Roche Pharmaceuticals, RCT Logic (formerly Clinical Trials Solutions), Sanofi-Aventis, Shire, Solvay Pharmaceuticals, Synthelabo, Wyeth-Ayerst Laboratories. MF also holds a patent for Sequential Parallel Comparison Design (SPCD), which is licensed by MGH to RCT Logic, and copyright for the MGH Cognitive \& Physical Functioning Questionnaire (CPFQ), Sexual Functioning Inventory (SFI), Antidepressant Treatment Response Questionnaire (ATRQ), Discontinuation-Emergent Signs \& Symptoms (DESS), and SAFER; Lippincott, Williams \& Wilkins; Wolkers Kluwer; World Scientific Publishing.

Ethical approval: This study obtained IRB approval from the Partners Human Research Committee (protocol No 2011-P-002314). No informed consent was required (this project was a retrospective study involving thousands of patients and multiple years of data, so consent could not feasibly be obtained from all subjects).

Data sharing: Additional data can be found in the supplementary figures and tables on bmj.com.

1 Roden DM. Drug-induced prolongation of the QT interval. N Engl J Med 2004;350:1013-22.

2 Van Noord C, Eijgelsheim M, Stricker BH. Drug-and non-drug-associated QT interval prolongation. Br J Clin Pharmacol 2010;70:16-23.

3 Arizona Center for Education and Research on Therapeutics. QT drug lists by risk group. www.azcert.org/medical-pros/drug-lists/drug-lists.cfm (accessed March 2012).

4 Glassman AH, Bigger JT Jr. Antipsychotic drugs: prolonged QTc interval, torsade de pointes, and sudden death. Am J Psychiatry 2001;158:1774-82.

5 Sala M, Lazzaretti M, De Vidovich G, Caverzasi E, Barale F, d'Allio G, et al. Electrophysiological changes of cardiac function during antidepressant treatment. Ther Adv Cardiovasc Dis 2009;3:29-43.

6 FDA Drug Safety Communication: Abnormal heart rhythms associated with high doses of Celexa (citalopram hydrobromide). MedWatch, 2011. www.fda.gov/Drugs/DrugSafety/ ucm269086.htm.

7 FDA Drug Safety Communication: Revised recommendations for Celexa (citalopram hydrobromide) related to a potential risk of abnormal heart rhythms with high doses. MedWatch, 2011. www.fda.gov/Drugs/DrugSafety/ucm297391.htm.

8 The use of medicines in the United States: review of 2011. IMS Institute for Healthcare Informatics, 2012.

9 Willis CD, McNeil JJ, Cameron PA, Phillips LE. Monitoring drug safety with registries: useful components of postmarketing pharmacovigilance systems. $J$ Clin Epidemiol 2012;65:121-5.

10 Brownstein JS, Murphy SN, Goldfine AB, Grant RW, Sordo M, Gainer V, et al. Rapid identification of myocardial infarction risk associated with diabetes medications using electronic medical records. Diabetes Care 2010;33:526-31.

11 Castro VM, Gallagher PJ, Clements CC, Murphy SN, Gainer VS, Fava M, et al. Incident user cohort study of risk for gastrointestinal bleed and stroke in individuals with major depressive disorder treated with antidepressants. BMJ Open 2012;2:e000544. doi:10. 1136/bmjopen-2011-000544.

12 Tatonetti NP, Denny JC, Murphy SN, Fernald GH, Krishnan G, Castro V, et al. Detecting drug interactions from adverse-event reports: interaction between paroxetine and pravastatin increases blood glucose levels. Clin Pharmacol Ther 2011;90:133-42. 
13 Murphy SN, Mendis M, Hackett K, Kuttan R, Pan W, Phillips LC, et al. Architecture of the open-source clinical research chart from Informatics for Integrating Biology and the Bedside. AMIA Annu Symp Proc 2007:548-52.

14 Murphy S, Churchill S, Bry L, Chueh H, Weiss S, Lazarus R, et al. Instrumenting the health care enterprise for discovery research in the genomic era. Genome Res 2009;19:1675-81.

15 Murphy SN, Weber G, Mendis M, Gainer V, Chueh HC, Churchill S, et al. Serving the enterprise and beyond with informatics for integrating biology and the bedside (i2b2). $J$ Am Med Inform Assoc 2010;17:124-30.

16 Tyl B, Azzam S, Blanco N, Wheeler W. Improvement and limitation of the reliability of automated QT measurement by recent algorithms. J Electrocardiol 2011;44:320-5.

17 Charlson ME, Pompei P, Ales KL, MacKenzie CR. A new method of classifying prognostic comorbidity in longitudinal studies: development and validation. J Chronic Dis 1987;40:373-83.

18 Charlson M, Szatrowski TP, Peterson J, Gold J. Validation of a combined comorbidity index. J Clin Epidemiol 1994;47:1245-51.

19 Points to consider: The assessment of the potential for QT interval prolongation by non-cardiovascular medicinal products. The European Agency for the Evaluation of Medicinal Products: Human Medicines Evaluation Unit; 1997:986-96.

20 Leonard CE, Bilker WB, Newcomb C, Kimmel SE, Hennessy S. Antidepressants and the risk of sudden cardiac death and ventricular arrhythmia. Pharmacoepidemiol Drug Saf 2011;20:903-13.

21 Kiev A, Masco HL, Wenger TL, Johnston JA, Batey SR, Holloman LC. The cardiovascular effects of bupropion and nortriptyline in depressed outpatients. Ann Clin Psychiatry 1994;6:107-15.

22 Wenger TL, Cohn JB, Bustrack J. Comparison of the effects of bupropion and amitriptyline on cardiac conduction in depressed patients. J Clin Psychiatry 1983;44:174-5.

23 Jeon SH, Jaekal J, Lee SH, Choi BH, Kim KS, Jeong HS, et al. Effects of nortriptyline on QT prolongation: a safety pharmacology study. Hum Exp Toxicol 2011;30:1649-56.

24 Hennessy S, Bilker WB, Knauss JS, Margolis DJ, Kimmel SE, Reynolds RF, et al. Cardiac arrest and ventricular arrhythmia in patients taking antipsychotic drugs: cohort study using administrative data. BMJ 2002;325:1070.
25 Enger C, Cali C, Walker AM. Serious ventricular arrhythmias among users of cisapride and other QT-prolonging agents in the United States. Pharmacoepidemiol Drug Saf 2002;11:477-86.

26 Kornbluh R, Papakostas GI, Petersen T, Neault NB, Nierenberg AA, Rosenbaum JF, et al. A survey of prescribing preferences in the treatment of refractory depression: recent trends. Psychopharmacol Bull 2001;35:150-6.

27 Sanguinetti MC, Jiang C, Curran ME, Keating MT. A mechanistic link between an inherited and an acquired cardiac arrhythmia: HERG encodes the IKr potassium channel. Cell 1995;81:299-307.

28 Hennessy S, Leonard CE, Palumbo CM, Bilker WB, Newcomb C, Kimmel SE. Diagnostic codes for sudden cardiac death and ventricular arrhythmia functioned poorly to identify outpatient events in EPIC's General Practice Research Database. Pharmacoepidemiol Drug Saf 2008;17:1131-6.

29 Glassman AH, O'Connor CM, Califf RM, Swedberg K, Schwartz P, Bigger JT Jr, et al. Sertraline treatment of major depression in patients with acute MI or unstable angina. JAMA 2002;288:701-9.

30 O'Connor CM, Jiang W, Kuchibhatla M, Silva SG, Cuffe MS, Callwood DD, et al. Safety and efficacy of sertraline for depression in patients with heart failure: results of the SADHART-CHF (Sertraline Against Depression and Heart Disease in Chronic Heart Failure) trial. J Am Coll Cardiol 2010;56:692-9.

Accepted: 18 December 2012

\section{Cite this as: BMJ 2013;346:f288}

This is an open-access article distributed under the terms of the Creative Commons Attribution Non-commercial License, which permits use, distribution, and reproduction in any medium, provided the original work is properly cited, the use is non commercial and is otherwise in compliance with the license. See: http://creativecommons.org/licenses/bync/2.0/ and http://creativecommons.org/licenses/by-nc/2.0/legalcode. 


\section{Tables}

Table 1| Demographics and clinical features of study cohort of 38397 adult patients with an electrocardiogram recorded 14-90 days after prescription of antidepressant or methadone between February 1990 and August 2011. Values are numbers (percentages) of patients unless stated otherwise

Value

Demographics

Mean (SD) age (years)

Female

Race/ethnicity†:

White 306

African American

Hispanic 2987

Asian

Other

Health Insurance

\begin{tabular}{|c|c|c|}
\hline Public & $20052(52.2)$ & $<0.001$ \\
\hline Private & $15851(41.3)$ & \\
\hline Other/unknown & $2494(6.5)$ & \\
\hline Median (IQR) year of electrocardiogram & 2007 (2004-09) & $<0.001$ \\
\hline \multicolumn{3}{|l|}{ Comorbidities (lifetime history) } \\
\hline Major depression & $10930(28.5)$ & $<0.001$ \\
\hline Myocardial infarction & $10391(27.1)$ & $<0.001$ \\
\hline Ventricular arrhythmia & $7968(20.8)$ & $<0.001$ \\
\hline Hypertension & $28145(73.3)$ & $<0.001$ \\
\hline Hyperlipidaemia & $23384(60.9)$ & $<0.001$ \\
\hline Median (IQR) age adjusted Charlson comorbidity index & $6(3-10)$ & $<0.001$ \\
\hline \multicolumn{3}{|l|}{ Pharmacotherapy } \\
\hline \multicolumn{2}{|l|}{ SSRIs: } & \multirow[t]{15}{*}{$<0.001$} \\
\hline Citalopram & $9777(25.5)$ & \\
\hline Escitalopram & $2364(6.2)$ & \\
\hline Fluoxetine & $5721(14.9)$ & \\
\hline Paroxetine & $4205(11.0)$ & \\
\hline Sertraline & $6293(16.4)$ & \\
\hline \multicolumn{2}{|l|}{ Other antidepressants: } & \\
\hline Duloxetine & $1912(5.0)$ & \\
\hline Venlafaxine & $2480(6.5)$ & \\
\hline Bupropion & $5064(13.2)$ & \\
\hline Mirtazapine & $3487(9.1)$ & \\
\hline Amitriptyline & $4228(11.0)$ & \\
\hline Nortriptyline & $1978(5.2)$ & \\
\hline \multicolumn{2}{|l|}{ Opiate antagonist: } & \\
\hline Methadone (known QTc-prolonging agent) & $2255(5.9)$ & \\
\hline
\end{tabular}

Methadone (known QTc-prolonging agent)

IQR=interquartile range. SSRI= selective serotonin reuptake inhibitor

${ }^{*}$ Corrected QT (QTc) interval recorded on electrocardiogram.

†Race and ethnicity are collected using a single field in the electronic health record, so subjects who identify as Hispanic are not further characterised. 
Table 2| Overall effect of dose of antidepressant or methadone on corrected QT (QTC) interval 14-90 days after drug prescription in cohort of 38397 adult patients. Results are beta values (SE) from linear regression analysis

\begin{tabular}{|c|c|c|}
\hline Drug & Unadjusted model & Adjusted model $†$ \\
\hline \multicolumn{3}{|l|}{ SSRIs: } \\
\hline Citalopram & $0.02(0.04)$ & $0.10(0.04)^{\star *}$ \\
\hline Escitalopram & $0.60(0.15)^{\star \star *}$ & $0.58(0.15)^{\star \star \star}$ \\
\hline Fluoxetine & $-0.01(0.03)$ & $0.07(0.03)$ \\
\hline Paroxetine & $-0.07(0.07)$ & $0.03(0.07)$ \\
\hline Sertraline & $-0.02(0.01)$ & $0.01(0.01)$ \\
\hline \multicolumn{3}{|c|}{ Other antidepressants: } \\
\hline Amitriptyline & $0.10(0.03)^{\star \star \star}$ & $0.11(0.03)^{\star \star *}$ \\
\hline Bupropion & $-0.03(0.01)^{* * *}$ & $-0.02(0.01)^{*}$ \\
\hline Duloxetine & $-0.00(0.05)$ & $0.02(0.05)$ \\
\hline Mirtazapine & $-0.22(0.08)^{* *}$ & $-0.13(0.08)$ \\
\hline Nortriptyline & $-0.01(0.04)$ & $0.04(0.04)$ \\
\hline Venlafaxine & $0.01(0.01)$ & $0.01(0.01)$ \\
\hline \multicolumn{3}{|l|}{ Positive control: } \\
\hline Methadone & $0.27(0.06)^{\star \star \star}$ & $0.30(0.06)^{\star \star *}$ \\
\hline
\end{tabular}

$\mathrm{SSRI}=$ selective serotonin reuptake inhibitor.

Statistical significance at ${ }^{*} \mathrm{a}<0.05,{ }^{* *} \mathrm{a}<0.01,{ }^{* * *} \mathrm{a}<0.001$.

†Model is adjusted for age; sex; race; type of health insurance; year of electrocardiogram (for QTc); history of major depression, myocardial infarction, ventricular arrhythmia, hypertension, or hyperlipidaemia; and log transformed, age adjusted Charlson comorbidity score. 
Table 3| Post hoc comparison of doses of antidepressants or methadone with an overall significant effect on corrected QT (QTC) interval in cohort of 38397 adult patients. Results are beta values (SE) from linear regression analysis

\begin{tabular}{|c|c|c|}
\hline Drug dose (mg) & Unadjusted model & Adjusted model† \\
\hline \multicolumn{3}{|l|}{ SSRIs: } \\
\hline \multicolumn{3}{|l|}{ Citalopram: } \\
\hline $10 \rightarrow 20$ & $11.3(1.6)^{\star \star *}$ & $9.8(1.6)^{\star \star \star}$ \\
\hline $20 \rightarrow 30$ & $-4.0(2.8)$ & $-0.9(2.7)$ \\
\hline $30 \rightarrow 40$ & $0.0(2.9)$ & $-0.7(2.8)$ \\
\hline $40 \rightarrow 60$ & $4.6(2.1)^{\star}$ & $6.1(2.1)^{\star \star}$ \\
\hline \multicolumn{3}{|l|}{ Escitalopram: } \\
\hline $5 \rightarrow 10$ & $11.7(4.5)^{\star \star}$ & $11.0(4.5)^{\star}$ \\
\hline $10 \rightarrow 20$ & $4.7(1.6)^{* *}$ & $4.7(1.6)^{\star \star}$ \\
\hline \multicolumn{3}{|c|}{ Other antidepressants: } \\
\hline \multicolumn{3}{|l|}{ Amitriptyline: } \\
\hline $10 \rightarrow 20$ & $-1.3(3.3)$ & $-0.8(3.3)$ \\
\hline $20 \rightarrow 25$ & $6.3(3.2)$ & $5.30(3.3)$ \\
\hline $25 \rightarrow 50$ & $3.0(1.4)^{\star}$ & $3.4(1.4)^{\star}$ \\
\hline $50 \rightarrow 75$ & $-3.4(3.4)$ & $-2.0(3.3)$ \\
\hline $75 \rightarrow 100$ & $3.0(4.1)$ & $1.9(4.1)$ \\
\hline \multicolumn{3}{|l|}{ Bupropion } \\
\hline $75 \rightarrow 100$ & $-1.3(2.1)$ & $0.0(2.1)$ \\
\hline $100 \rightarrow 150$ & $-1.5(1.3)$ & $-3.3(2.2)$ \\
\hline $150 \rightarrow 200$ & $-3.1(2.1)$ & $-1.8(2.1)$ \\
\hline $200 \rightarrow 300$ & $-1.2(2.6)$ & $-0.3(2.5)$ \\
\hline \multicolumn{3}{|l|}{ Positive control: } \\
\hline \multicolumn{3}{|l|}{ Methadone } \\
\hline $3 \rightarrow 5$ & $-3.0(2.5)$ & $9.6(6.5)$ \\
\hline $5 \rightarrow 10$ & $3.0(2.4)$ & $2.4(2.5)$ \\
\hline $10 \rightarrow 15$ & $-8.1(5.1)$ & $-7.2(5.1)$ \\
\hline $15 \rightarrow 20$ & $4.7(5.6)$ & $4.5(5.6)$ \\
\hline $20 \rightarrow 30$ & $10.3(5.6)$ & $10.1(5.6)$ \\
\hline $30 \rightarrow 40$ & $0.0(5.3)$ & $0.9(5.3)$ \\
\hline $40 \rightarrow 50$ & $4.7(3.9)$ & $3.2(3.9)$ \\
\hline
\end{tabular}

SSRI= selective serotonin reuptake inhibitor.

Statistical significance at ${ }^{*} \mathrm{a}<0.05,{ }^{* \star} \mathrm{a}<0.01,{ }^{* \star *} \mathrm{a}<0.001$.

†Model is adjusted for age; sex; race; type of health insurance; year of electrocardiogram; history of major depression, myocardial infarction, ventricular arrhythmia, hypertension, or hyperlipidaemia; and log transformed, age adjusted Charlson comorbidity score. 
Table 4| Results of within-patient change in corrected QT (QTc) interval after escalating doses of antidepressants or methadone for drugs with a significant dose association

Dose changes (mg) No of patients Mean (SE) change in QTc (ms) Paired $t$ statistic Citalopram:

\begin{tabular}{|c|c|c|c|}
\hline $10 \rightarrow 20$ & 59 & $7.8(3.6)$ & $2.1^{\star \star}$ \\
\hline $10 \rightarrow 40$ & 17 & $16.5(9.1)$ & 1.8 \\
\hline $20 \rightarrow 30$ & 17 & $6.9(6.1)$ & 1.1 \\
\hline $20 \rightarrow 40$ & 107 & $10.3(4.0)$ & $2.6^{*}$ \\
\hline $20 \rightarrow 60$ & 30 & $3.8(5.1)$ & 0.8 \\
\hline $40 \rightarrow 60$ & 14 & $5.4(11.1)$ & 0.5 \\
\hline \multicolumn{4}{|c|}{ Escitalopram: } \\
\hline $10 \rightarrow 20$ & 37 & $6.4(7.6)$ & 0.8 \\
\hline \multicolumn{4}{|c|}{ Amitriptyline: } \\
\hline $10 \rightarrow 20$ & 10 & $-3.6(7.3)$ & -0.5 \\
\hline $10 \rightarrow 25$ & 16 & $-3.2(7.1)$ & -0.4 \\
\hline $25 \rightarrow 50$ & 28 & $7.1(5.2)$ & 1.4 \\
\hline \multicolumn{4}{|l|}{ Bupropion: } \\
\hline $75 \rightarrow 150$ & 18 & $3.1(6.8)$ & 0.5 \\
\hline $100 \rightarrow 150$ & 37 & $-0.8(6.5)$ & -0.1 \\
\hline $100 \rightarrow 200$ & 13 & $-19.2(8.7)$ & $-2.2^{*}$ \\
\hline $150 \rightarrow 200$ & 10 & $-0.2(15.9)$ & 0.0 \\
\hline $150 \rightarrow 300$ & 13 & $1.7(5.4)$ & 0.3 \\
\hline \multicolumn{4}{|l|}{ Methadone: } \\
\hline $5 \rightarrow 10$ & 18 & $3.2(9.5)$ & 0.3 \\
\hline $10 \rightarrow 20$ & 12 & $-0.6(5.3)$ & -0.1 \\
\hline $10 \rightarrow 40$ & 14 & $-3.1(9.1)$ & -0.3 \\
\hline
\end{tabular}




\section{Figure}
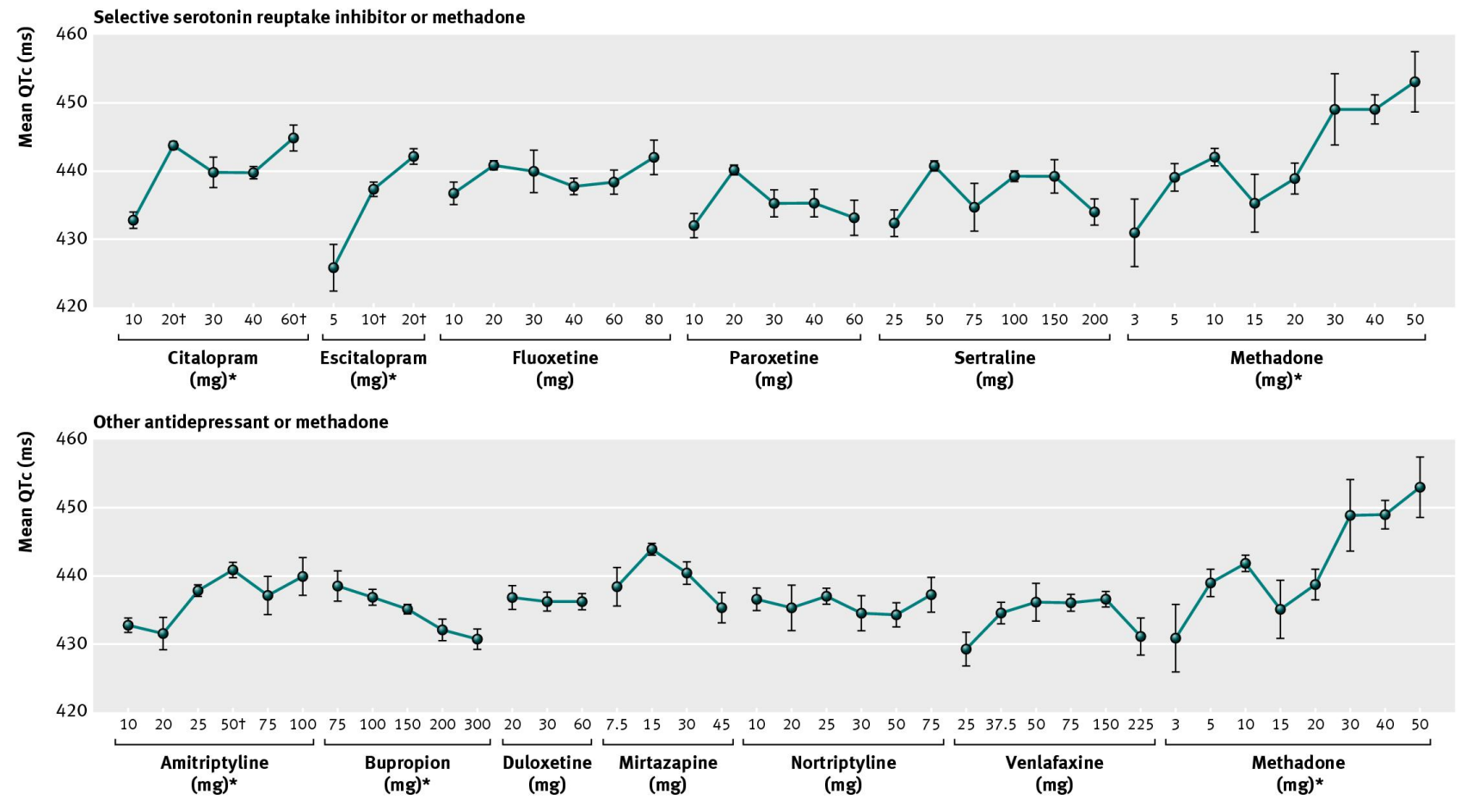

* Dose a significant predictor of QTc in fully adjusted linear models at $a=0.05$

+ QTc at specified dose is significantly different from that at prior dose in fully adjusted linear models at $a=0.05$

Mean (SD) corrected QT (QTc) interval recorded on electrocardiogram 14-90 days after prescription of antidepressant or methadone, by drug dose 